\title{
Screening for Type 2 Diabetes - The ADDITION Netherlands Study
}

\author{
Guy EHM Rutten ${ }^{1}$ and Paul GH Janssen² \\ 1. Professor of Diabetology; 2. General Practitioner, Julius Centre for Health Sciences and Primary Care, University Medical Centre Utrecht
}

DOI:10.17925/EE.2009.05.00.32

\begin{abstract}
To investigate whether early treatment of screening-detected diabetic patients is beneficial, the Anglo-Danish-Dutch Study of Intensive Treatment in People with Screen-Detected Diabetes in Primary Care (ADDITION) has been initiated. A total of 56,978 subjects 50-70 years of age without diabetes from 79 general practices in The Netherlands were invited to participate in a stepwise screening programme. Five hundred and eighty-six participants (1.0\%) were diagnosed with type 2 diabetes. The score on the initial risk questionnaire was higher if glucose metabolism was more disturbed. The yield of screening varied widely between practices. A lower yield was not associated with an appropriate practice organisation regarding diabetes care, nor with a speciality of the GP in diabetes. Opportunistic screening in general practice seems preferable to population-based screening. Intensified multifactorial treatment of patients with screening-detected type 2 diabetes in general practice reduced the cardiovascular risk factor levels significantly after just one year without worsening healthrelated quality of life. After three years of follow-up, screened participants without diabetes but with an elevated risk score had comparable cardiovascular event rates to patients with diabetes. Screened individuals without diabetes are at risk of lacking optimal control of cardiovascular risk factors.
\end{abstract}

\section{Keywords}

Cardiovascular disease, healthcare delivery, impaired glucose tolerance, impaired fasting glucose, primary care, screening, type 2 diabetes

Disclosure and Acknowledgements: The authors are grateful to Professor Ronald Stolk and Dr Kees Gorter for their substantive contributions to the article. The ADDITION Netherlands study is made possible by unrestricted grants from Novo Nordisk, GlaxoSmithKline and Merck.

Received: 6 May 2009 Accepted: 16 June 2009

Correspondence: Guy Rutten, Professor of Diabetology in Primary Care, University Medical Centre Utrecht, Julius Centre for Health Sciences and Primary Care, PO Box 85500, 3508 GA Utrecht, The Netherlands. E: G.E.H.M.Rutten@umcutrecht.nl

The prevalence of type 2 diabetes is rapidly increasing. ${ }^{1}$ Most people are asymptomatic at diagnosis and the assumption is made that early diagnosis and treatment of type 2 diabetes will be beneficial, although definitive evidence is lacking. ${ }^{2}$ The American Diabetes Association stated that there is sufficient indirect evidence to justify opportunistic screening in a clinical setting. ${ }^{3}$ Recently, the International Diabetes Federation also recommended opportunistic screening. ${ }^{4}$ In The Netherlands, the Dutch College of General Practitioners recommends opportunistic screening (case-finding) for diabetes in people at risk of type 2 diabetes. ${ }^{5}$ To investigate whether early treatment of patients with screening-detected diabetes is beneficial, the Anglo-DanishDutch Study of Intensive Treatment in People with Screen-Detected Diabetes in Primary Care (ADDITION) has been initiated. ${ }^{6}$

ADDITION is a multicentre randomised controlled trial that consists of a screening study and a subsequent intervention trial with a follow-up of five years. In the screening study, the feasibility of identifying persons with type 2 diabetes is evaluated. In the intervention study (a single-blind, multipractice trial with practicelevel randomisation), the effects of routine care in general practice according to national guidelines are compared with those of an intensified, multifactorial treatment on cardiovascular mortality and morbidity (non-fatal myocardial infarction and non-fatal stroke), revascularisation and amputations.
This article describes the results of population-based screening for diabetes in The Netherlands and the associations of the yield of the screening with characteristics of general practitioners (GPS) and practices. Furthermore, we report on the one-year results of the intervention trial. Finally, we followed people in different glucose regulation categories over three years regarding the extent of healthcare utilisation and the risk of cardiovascular disease (CVD).

\section{Patients and Methods}

\section{Patients}

In The Netherlands, the entire population is registered with a GP. Therefore, the screened population may be considered a representative sample of the full population. All 56,978 patients, who were $50-70$ years of age and not known to have diabetes at study entry, from 79 general practices in the south-western region of The Netherlands were invited to participate in the screening programme.

Exclusion criteria for the intervention study were: any contraindications or history of major intolerance to any of the drugs used in the study; a history of alcoholism, drug abuse, psychosis, personality disorder or another emotional, psychological or intellectual problem that would likely invalidate informed consent or limit the ability to comply with the protocol requirements; and those being treated for a malignant disease or otherwise having a poor prognosis. 
The study was approved by the medical ethical committee of the University Medical Centre Utrecht. Participants gave written informed consent.

\section{General Practitioners and Practices}

A total of 106 GPS in 79 practices participated in the study; 41 practices participated in the first screening procedure and 38 in the second. Forty-two practices were randomised to the routine care group and 37 to the intensified treatment group.

\section{Screening Programme}

Two stepwise screening procedures were performed. The first procedure (May 2002 - January 2003) consisted of four steps: a selfcompleted risk questionnaire, random glucose measurement (RBG), fasting glucose measurement (FBG) and an oral glucose tolerance test (OGTT). In the second procedure (July 2003 - April 2004), no RBG was performed. The initial questionnaire contained questions about age, gender, body mass index (BMI), family history of diabetes, frequent thirst, the use of antihypertensive medication, shortness of breath, claudication and cycling.? People who scored above a pre-defined threshold were invited to undergo subsequent diagnostic glucose testing. Participants were classified according to the 1999 World Health Organization (WHO) criteria. ${ }^{8}$

\section{Intensified Multifactorial Treatment}

The intensified treatment was according to the common ADDITION treatment protocol, which is characterised by intensive treatment of glucose, blood pressure and lipids and structured lifestyle education (dietary modification, weight loss, increased physical activity, smoking cessation and improving adherence to medication). The glycated haemoglobin $\left(\mathrm{HbA}_{1 \mathrm{c}}\right)$ level should be kept under $7.0 \%$. Intensification of glucose-lowering therapy should be initiated while $\mathrm{HbA}_{1 \mathrm{c}}$ is $>6.5 \%$. If $\mathrm{HbA}_{1 \mathrm{C}}$ remains above $7.0 \%$ with oral agents, insulin therapy should be initiated. Antihypertensive agents were prescribed if blood pressure was $>120 / 80 \mathrm{mmHg}$ (systolic or diastolic). Angiotensinconverting enzyme (ACE) inhibitors were the initial treatment. In the case of side effects, an angiotensin-II receptor antagonist was prescribed. If blood pressure was $>135 / 85 \mathrm{mmHg}$, the dose had to be increased, and thiazides, calcium channel blockers or beta-blockers were added using a stepwise approach. Treatment with a statin was indicated if cholesterol was $>5.0 \mathrm{mmol} / /$ or $>4.5 \mathrm{mmol} / /$ in patients without or with a known history of CVD, respectively. The dose of statin was increased up to maximum if cholesterol remained above threshold. In 2003, the protocol changed: all participants with cholesterol $>3.5 \mathrm{mmol} / \mathrm{l}$ were treated with a statin. Acetylsalicylic acid $80 \mathrm{mg}$ was given to patients treated with antihypertensive agents. In the routine care group, the treatment goals according to the Dutch guidelines were less ambitious. The target value for $\mathrm{HbA}_{1 \mathrm{c}}$ was $<7.0 \%$; however, $\mathrm{HbA}_{1 \mathrm{c}}$ levels between 7.0 and $8.5 \%$ were described as acceptable. Lipid-lowering drugs were initiated if participants without a known history of CVD had a greater than 25\% risk of developing a coronary disease within 10 years, while all those with previous CVD were treated with a statin. If blood pressure was $>150 / 85 \mathrm{mmHg}$, treatment was recommended. Detailed instructions about providing lifestyle education were not given.

\section{Three-year Follow-up of Persons in Different Glucose Regulation Categories}

All persons with a risk score above threshold from 24 practices, screened from May to October 2002, were followed over three years to determine the extent of healthcare utilisation and to evaluate the occurrence of CVD. Patients with diabetes from practices in the intervention arm of the ADDITION study were excluded. We collected data at baseline and after three years from 354 subjects in four categories of glucose regulation: type 2 diabetes $(n=64)$, impaired glucose tolerance (IGT) ( $n=62)$, impaired fasting glucose (IFG) $(n=86)$ and normal glucose tolerance (NGT) but with an elevated risk score $(n=142)$.

\section{Measurements}

Capillary blood glucose values were determined using a HemoCue B-Glucose Analyzer based on the glucosedehydrogenase method. Plasma glucose was measured using a peroxidase method. $\mathrm{HbA}_{1 \mathrm{c}}$ was assessed with high-performance liquid chromatography (160 A. Menarini Diagnostics). Cholesterol, high-density lipoprotein (HDL) cholesterol and triglycerides were determined using enzymatic techniques (LX20 Beckman). The Short Form (SF)-36 measures general health, vitality, mental health, physical functioning, limitations due to physical difficulties (role physical), bodily pain, social functioning and limitations due to emotional difficulties (role emotional). For each dimension, scores range from 0 (worst health) to 100 (best health).

\section{Data Analyses}

The association between risk score and different glucose tolerance categories was analysed using linear regression. Associations of the yield of screening with GP and practice characteristics were studied using multiple regression analysis. An independent variable in the model was the number of detected patients with diabetes per practice after adjustment for practice size and age distribution (standardised practice). Changes in biomedical variables after one year were compared between both groups using generalised estimating equations adjusting for age, gender, baseline values and clustering at practice level. Analyses were based on intention-totreat. SF-36 scores at the end of study were analysed using analysis of co-variance (ANCOVA), with age, gender, and baseline values as co-variates to adjust for differences between treatment groups at randomisation. Paired-sample t-tests were used to compare baseline with follow-up within each treatment group. Gender and age at baseline were compared between the different diagnostic categories using chi-square test for dichotomous variables and analysis of variance (ANOVA) for continuous variables, respectively. Healthcare encounters were analysed using the Mann-Whitney test. Differences between diagnostic categories in the use of medication were examined with the chi-square test. Cardiovascular events and death were calculated per diagnostic category per 1,000 person-years of follow-up. Cox regression analysis with age and gender entered as co-variates in the model was used to examine whether the diagnostic categories have different risks regarding mortality and cardiovascular morbidity. The data were analysed applying the SPSS statistical package (version 15.0). A p-value $<0.05$ was considered significant.

\section{Results}

The screening algorithm and yields are presented in Figures 1 and 2 . Eventually, 586 participants (1.0\%) were diagnosed with type 2 diabetes (four-step procedure: 285 people; three-step procedure: 301 people). Impaired glucose regulation was diagnosed in 1,011 participants $(1.8 \%){ }^{9}$

Risk scores ( \pm standard deviation [SD]) of persons with NGT, IFG, IGT, epidemiological diabetes and type 2 diabetes in the four-step 


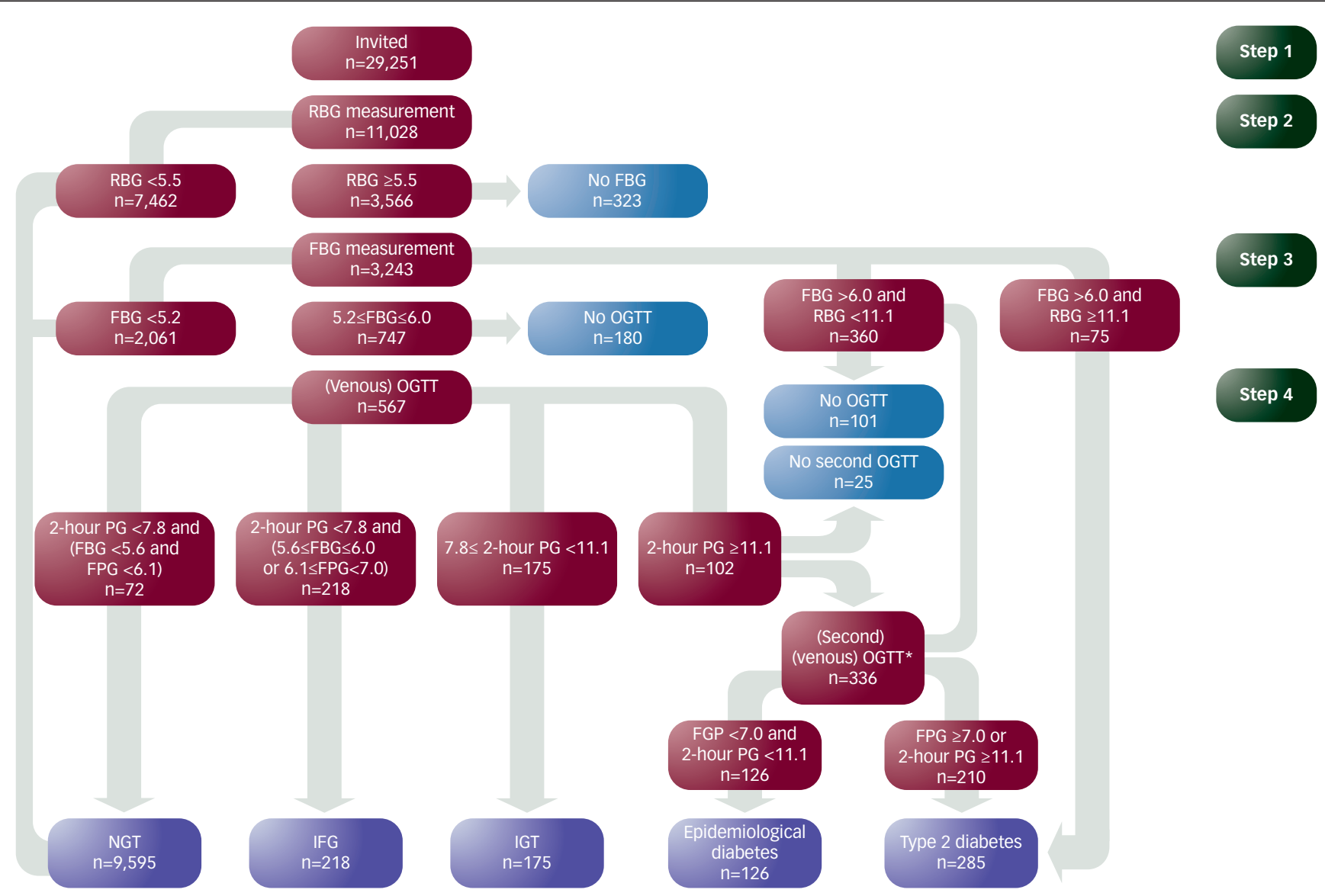

Blue boxes: patients who did not show up.

2-hour PG = 2-hour plasma glucose; epidemiological diabetes = one diabetic glucose value; FBG = fasting blood glucose; FPG = fasting plasma g/ucose; IFG = impaired fasting g/ucose; $I G T=$ impaired glucose tolerance: $N G T=$ normal glucose tolerance: $O G T=$ oral glucose tolerance test: $R G B=$ random blood glucose.

*Second OGTT for subjects with $5.2 \mathrm{mmol} / \mathrm{l} \leq F B G \leq 6.0 \mathrm{mmol} /$; first OGTT for those with FBG >6.0 mmol/l and RBG <11.1 mmol/l. Source: Janssen et al., $2007 .{ }^{9}$

\section{Figure 2: Outline of the Three-step Screening Procedure}

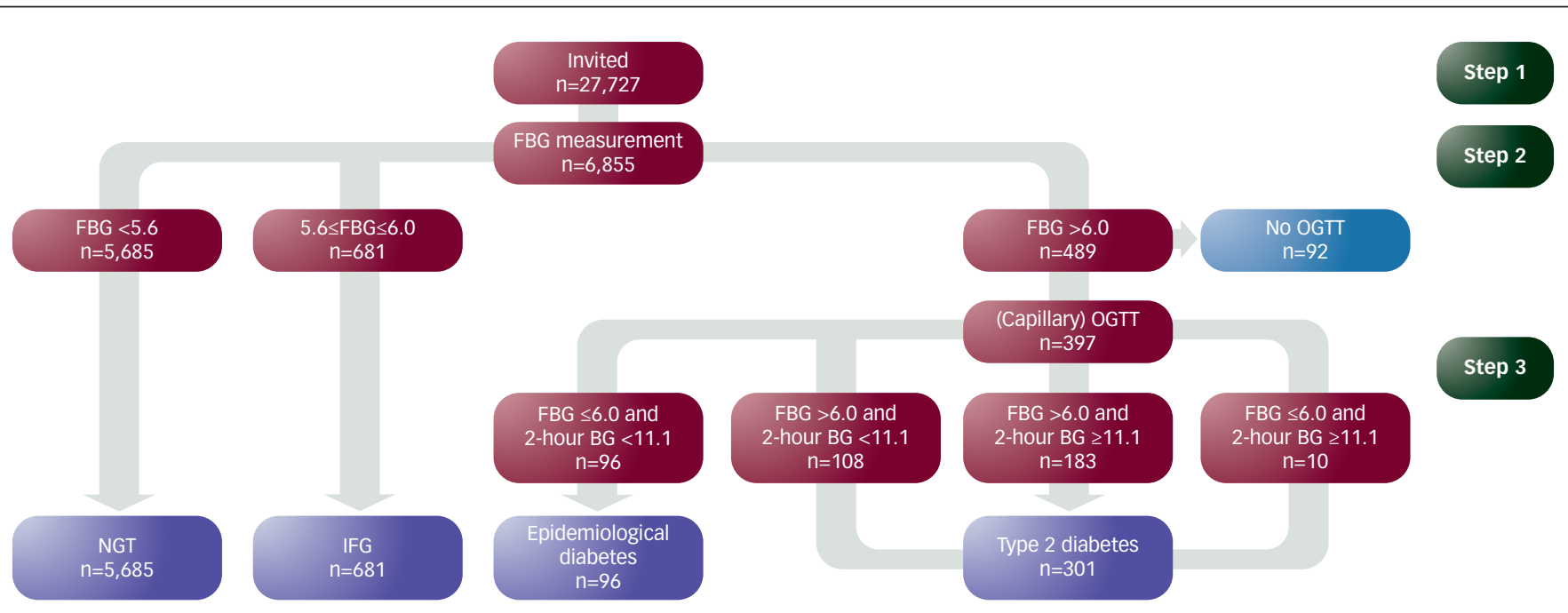

Blue box: patients who did not show up.

2-hour BG = 2-hour fasting plasma glucose; epidemiological diabetes = one diabetic g/ucose value; FBG = fasting blood glucose; IFG = impaired fasting g/ucose $N G T=$ normal glucose tolerance; OGTT = oral glucose tolerance test. Source: Janssen et al., 2007. ${ }^{\text {? }}$

procedure were $7.5 \pm 4.3,8.5 \pm 4.9,8.9 \pm 4.5,9.8 \pm 5.2$ and $10.5 \pm 5.0$, respectively. In the three-step procedure, the risk scores were $8.0 \pm 4.1$ for NGT, $9.2 \pm 4.2$ for IGT, $10.0 \pm 4.2$ for epidemiological diabetes and $10.3 \pm 4.7$ for type 2 diabetes. In both procedures the risk score proved to be higher if glucose metabolism was more disturbed ( $p$ for trend $<0.001$ ).
The yield of screening per practice varied widely. The number of screening-detected patients with diabetes per standardised practice ranged from 1.1 to 14.1 (mean 5.3, SD \pm 2.7 ). Higher age of the GP (regression coefficient $0.10,95 \%$ confidence interval $[\mathrm{Cl}]$ 0.01-0.19) and involvement of the practice assistant in diabetes care (regression coefficient $1.32,95 \% \mathrm{Cl} 0.21-2.43$ ) were independently associated 
with a higher yield, reflecting more undiagnosed patients with diabetes. In contrast, urban location (regression coefficient -2.50, 95\% $\mathrm{Cl}-3.63$ to -1.37 ) and co-operation with a diabetes nurse (regression coefficient $-1.32,95 \% \mathrm{Cl}-2.38$ to -0.05 ) were associated with a lower number of undiagnosed patients with diabetes. ${ }^{10}$

Cardiovascular risk profiles of people with IFG did not resemble the profiles of those with IGT or diabetes. The poorer the glycaemic control, the worse the levels of $\mathrm{BMI}$ and blood pressure. BMI was $27.3 \pm 4.4,29.5 \pm 5.7$ and $30.7 \pm 5.6$ in persons with IFG, IGT and diabetes, respectively ( $p$ for trend $<0.001$ ); systolic blood pressure was $150 \pm 25$, $161 \pm 24$ and $162 \pm 23 \mathrm{mmHg}$, respectively ( $p$ for trend $<0.001$ ); and diastolic blood pressure was $84 \pm 12,89 \pm 12$ and $90 \pm 11 \mathrm{mmHg}$, respectively ( $\mathrm{p}$ for trend $<0.001$ ). When $\mathrm{BMI}$ was higher, age- and gender-adjusted cardiovascular risk factors were even more adverse, especially in those with diabetes. ${ }^{11}$

The trial profile is shown in Figure 3. Of the 586 patients with screening-detected diabetes, 498 were included. A total of 255 subjects were assigned to intensified treatment and 243 to routine care. The two groups were well matched with respect to clinical, biochemical and behavourial characteristics, as well as use of cardiovascular medications and history of cardiovascular events (data not shown). Almost all cardiovascular risk factors improved to a significantly greater extent in the first year of treatment in the intensified care group compared with the routine care group (see Table 1). The most striking differences were found in the change of mean $\mathrm{BMI}(+0.2$ for routine care versus -1.4 for intensified care), systolic blood pressure (-19 versus $-33 \mathrm{mmHg})$, diastolic blood pressure $(-7$ versus $-12 \mathrm{mmHg})$, cholesterol $(-0.5$ versus $-1.2 \mathrm{mmol} / \mathrm{l})$ and LDL cholesterol ( -0.5 versus $-1.0 \mathrm{mmol} / \mathrm{l})$. One hypoglycaemic event requiring assistance occurred in the intervention group. ${ }^{12}$

\section{Against this background, one could argue \\ that opportunistic screening in high-risk \\ patients in general practice is likely to be \\ more suitable for detecting unknown \\ diabetes than population-based screening.}

SF-36 scores were not significantly different between the two treatment groups at the end of the study (see Table 2). Within both treatment groups, general health, vitality and mental health - and in the intensively treated group also physical functioning - improved significantly during follow-up. Scores on social functioning decreased equally in both groups.

In the separate three-year follow-up study it was shown that all categories of cardiovascular medication were prescribed more frequently after the screening in all screened people. The strongest increase was found in patients with screening-detected diabetes. The number of practice visits was higher in patients with diabetes compared with those in the other categories. Glucose, lipids and blood pressure were measured most frequently in patients with diabetes. The numbers of cardiovascular events in subjects with NGT, IFG, IGT and diabetes were $16.7,32.6,17.3$ and 15.7 per 1,000 personyears, respectively $(p=N S){ }^{13}$

\section{Figure 3: Trial Profile ${ }^{12}$}

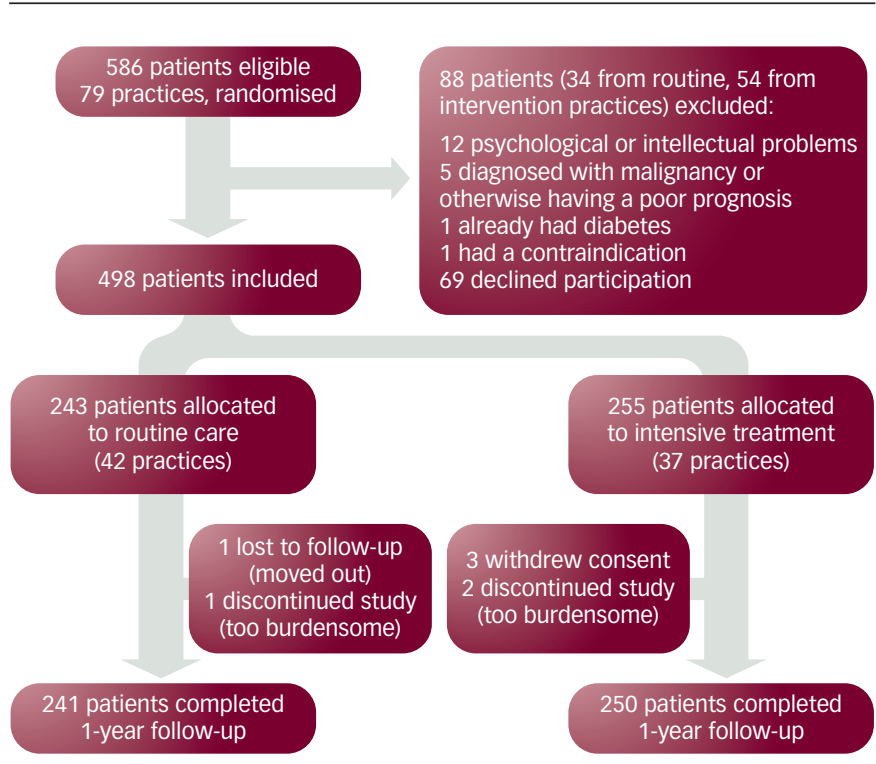

Source: Paul GH Janssen, Kees J Gorter, Ronald P Stolk and Guy EHM Rutten, Randomised controlled trial of intensive multifactorial treatment for cardiovascular risk in patients with screen-detected type 2 diabetes: 1-year data from the ADDITION Netherlands study, Br J Gen Pract, 2009;59:43-48.

\section{Discussion}

\section{Yield of Population-based Diabetes Screening}

The yield of our population-based diabetes screening programme was low, regardless of the number of steps in the procedure. In the mid1990s it was estimated that about half of all people with diabetes were undiagnosed, but we may conclude that this is no longer the case. Following the recommendations of the Dutch College of General Practitioners, screening for diabetes has become more common in Dutch general practices in the last decade, which is reflected in the strong increase in the prevalence of diabetes in the late 1990s. ${ }^{14}$ However, it should be emphasised that the true prevalence of undiagnosed diabetes remains unknown. Nevertheless, we may assume that the low yield of our screening programme was associated with the decreasing prevalence of undiagnosed diabetes. In ADDITION Denmark only $0.6 \%$ patients with screening-detected diabetes (between 40 and 70 years of age) were found, mainly due to a large drop-out prior to entry into the screening programme..$^{15}$

Against this background, one could argue that opportunistic screening in high-risk patients in general practice is likely to be more suitable for detecting unknown diabetes than population-based screening. Conducting a screening programme is expensive and timeconsuming. Opportunistic screening/case-finding incorporated into daily practice does not have these disadvantages. In The Netherlands, the necessary conditions for successful case-finding are fulfilled: the entire population is registered with a GP, and general practices are well organised with respect to daily diabetes care (involvement of practice assistants, practice nurses and diabetes nurses in diabetes care). Additionally, case-finding seems more applicable than other screening strategies in fulfilling the criterion that screening be a continuous process. ${ }^{16}$ Moreover, we found that increasing glucose intolerance is associated with a higher risk score. This reveals another possible advantage of case-finding: it may offer the possibility of detecting persons with different categories of impaired glucose regulation, most of whom already have an increased CVD risk. ${ }^{17}$ The considerable number of detected subjects with impaired glucose 
Table 1: Baseline Characteristics and Changes in Biomedical Variables After One Year in Both Treatment Groups

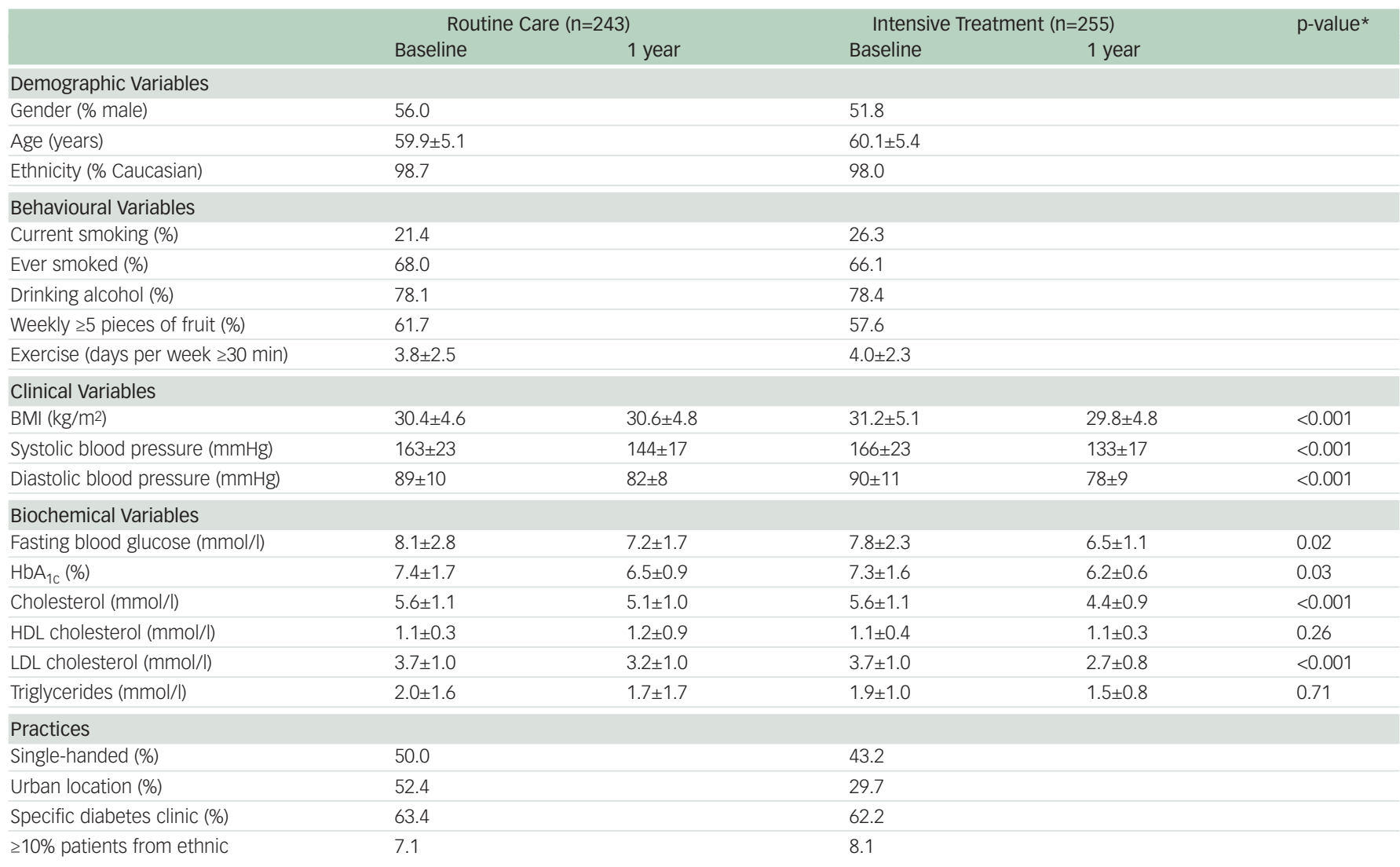

minority groups (\%)

Data are means \pm standard deviation (SD) unless otherwise indicated. *Comparison of changes in variables between treatment groups adjusted for age, gender, baseline value and clustering at practice level. $B M I=$ body mass index; $H b A_{1 C}=$ glycated haemoglobin; $H D L=$ high-density lipoprotein; $L D L=$ low-density lipoprotein. Source: Paul GH Janssen, Kees $J$ Gorter, Ronald P Stolk and Guy EHM Rutten, Randomised controlled trial of intensive multifactorial treatment for cardiovascular risk in patients with screen-detected type 2 diabetes: 1 -year data from the ADDITION Netherlands study, Br J Gen Pract, 2009;59:43-48.

Table 2: SF-36 Scores at Baseline and After One Year in Both Treatment Groups

\begin{tabular}{|c|c|c|c|c|c|}
\hline & \multicolumn{2}{|c|}{ Routine Care $(n=243)$} & \multicolumn{2}{|c|}{ Intensive Treatment $(n=255)$} & \multirow{2}{*}{$\begin{array}{l}\text { Difference Between Groups* } \\
\text { p-value }\end{array}$} \\
\hline & Baseline & One year & Baseline & One year & \\
\hline General health & $59.7 \pm 12.0$ & $64.4 \pm 18.1^{\dagger}$ & $59.1 \pm 11.5$ & $63.3 \pm 18.4^{\dagger}$ & 0.632 \\
\hline Vitality & $52.2 \pm 13.2$ & $67.1 \pm 18.4^{\dagger}$ & $49.3 \pm 14.4$ & $64.8 \pm 20.4^{\dagger}$ & 0.814 \\
\hline Mental health & $69.9 \pm 12.6$ & $79.0 \pm 15.6^{\dagger}$ & $68.4 \pm 13.3$ & $75.9 \pm 17.9+$ & 0.559 \\
\hline Physical functioning & $78.3 \pm 22.0$ & $78.1 \pm 23.2$ & $77.4 \pm 21.9$ & $80.1 \pm 21.2^{\dagger}$ & 0.218 \\
\hline Role physical & $84.9 \pm 30.0$ & $81.1 \pm 33.5$ & $82.8 \pm 31.4$ & $80.3 \pm 35.0$ & 0.930 \\
\hline Bodily pain & $84.7 \pm 20.7$ & $82.2 \pm 22.4$ & $80.8 \pm 22.1$ & $79.2 \pm 22.7$ & 0.970 \\
\hline Social functioning & $89.0 \pm 17.2$ & $85.7 \pm 19.2^{\dagger}$ & $87.9 \pm 20.0$ & $83.0 \pm 22.0 \dagger$ & 0.368 \\
\hline Role emotional & $85.4 \pm 32.4$ & $89.9 \pm 26.0$ & $88.2 \pm 28.6$ & $86.2 \pm 30.9$ & 0.254 \\
\hline
\end{tabular}

Data are means \pm standard deviation (SD). SF-36 = Short Form 36. *Differences between treatment groups at end of study, adjusted for age, gender and baseline value.

${ }^{\dagger}$ Difference between baseline and at end of study within group is significant. Source: Paul GH Janssen, Kees I Gorter, Ronald P Stolk and Guy EHM Rutten, Randomised controlled trial of intensive multifactorial treatment for cardiovascular risk in patients with screen-detected type 2 diabetes: 1-year data from the ADDITION Netherlands study, Br J Gen Pract, 2009;59:43-48.

regulation urges a proactive approach by healthcare providers in order to reduce the cardiovascular risk of their patients. ${ }^{18}$ Opportunistic screening in general practice for people with impaired glucose regulation could begin by filling in the risk questionnaire during practice visits.

\section{Intensified Multifactorial Treatment of} Screening-detected Type 2 Diabetic Patients

We found spectacular improvements in cardiovascular risk factor levels in the intensively treated group compared with the routine care group even after only one year of treatment. The final results of the ADDITION study, due in 2010, must be awaited before we can evaluate the effectiveness of early aggressive five-year treatment of all cardiovascular risk factors in people with screening-detected diabetes. ${ }^{19}$ In the Steno-2 Study, in which diabetic patients with microalbuminuria were included, the cardiovascular event rate was cut by half in the intensively treated group (mean follow-up 7.8 years). ${ }^{20,21}$

At the end of follow-up, SF-36 scores were similar for the two groups, suggesting no major detrimental impact on quality of life from the intensive intervention. Intensified multifactorial treatment of screening-detected patients with diabetes in general practice is likely to be feasible at the patient level. In a subset of patients in the ADDITION cohort $(n=196)$, psychological outcomes were examined. ${ }^{22}$ The intensively treated patients tended to report more distress and less self-efficacy in the first year after diagnosis than those who 
received routine care. However, two to three years after diagnosis the experienced psychological burden did not differ between the two groups. It may be assumed that distress in patients in the routine care group is delayed, since they were not confronted with rigorous treatment shortly after diagnosis. These findings suggest that even in the long run intensive treatment is feasible. It should be noted that patients in the intensified treatment group were treated by diabetes nurses who had enough time to motivate patients. Therefore, it may be disputable whether treatment targets as achieved in our trial are also achievable in daily practice. However, in many general practices specialised nurses are already involved in daily diabetes care, making the implementation of intensified treatment feasible.

\section{Persons with Impaired Glucose Tolerance and Impaired Fasting Glucose}

After three years of follow-up, screened subjects with an elevated risk score but without diabetes had comparable cardiovascular event rates to patients with diabetes. Screened people without diabetes are at risk of lacking optimal medical care in order to control for cardiovascular risk factors. They should not be reassured by the fact that they do not have diabetes. Since this sub-study of the ADDITION study was not prospectively designed to investigate differences in the occurrence of cardiovascular events between the glucose regulation categories, these findings should be interpreted with some caution.

\section{Implications for Policy and Practice}

Given its increasing prevalence, screening for diabetes and adverse cardiovascular risk has become a societal issue. In particular, obesity, physical inactivity, dietary habits and smoking may be considered as matters of public health. Preventing unhealthy lifestyle behaviours is not solely an issue to be managed in primary care. Nevertheless, GPS could play an important role.

The cardiovascular risk of people with impaired glucose regulation, especially when they are obese, should not be underestimated. In order to detect people with increased cardiovascular risk, a proactive, systematic opportunistic screening programme in all practices is needed. Practice nurses could play a pivotal role in the detection of increased diabetes and CVD risk using a simple risk questionnaire Intensified treatment of cardiovascular risk factors in general practice is feasible on the condition that it is delivered by specially trained and well-educated practice nurses in co-operation with the GP.

Given the substantial number of persons with IGT remaining unidentified without an OGTT, and taking into account their increased cardiovascular risk, ${ }^{23,24}$ the need to perform the OGTT in general practice should be re-considered. Using only the fasting glucose levels will fail to diagnose approximately $30 \%$ of people with diabetes, with an even greater failure rate in an older population..$^{25,26}$ Of the 285 people with diabetes detected in our four-step screening procedure, $36(12.6 \%)$ were found to have an initial non-diabetic fasting glucose value. This finding questions whether it is acceptable that screening for diabetes in general practice is based on fasting glucose testing alone. Without performing an OGTT, persons with IGT will not be recognised at all. since the first step in the deterioration of glucose homeostasis corresponds to a loss of post-prandial glycaemic control, ${ }^{27}$ detection of persons with type 2 diabetes will be delayed by measuring fasting glucose values only.

\section{Conclusions}

In order to identify patients with undiagnosed diabetes, opportunistic screening in general practice seems more appropriate than population-based screening. The increased cardiovascular risk of hyperglycaemia is notably present in overweight persons. Screening should not be targeted at hyperglycaemia alone but rather at cardiovascular risk profiles. Intensified multifactorial treatment of screening-detected type 2 diabetes patients in general practice will reduce the levels of cardiovascular risk factors without worsening health-related quality of life.

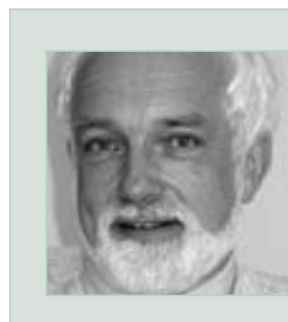

Guy EHM Rutten is a Professor of Diabetology in general practice at the Julius Centre for Health Sciences and Primary Care at the University Medical Centre Utrecht. He has been a general practioner (GP) since 1982 and continues to work as a GP for two days per week. His research activities focus on diabetes and cardiovascular complications and diabetes primary care. Professor Rutten has authored more than 100 original articles in national and international peer-reviewed journals. He chairs the Dutch General Practice Advisory Group and the European Association for the Study of Diabetes (EASD) Study Group on Primary Care Research in Diabetology, and was the first Editor in Chief of Primary Care Diabetes.

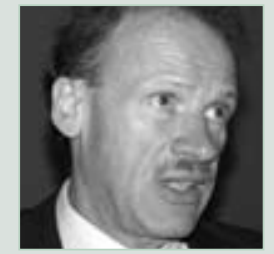

Paul GH Janssen is a General Practitioner in Baambrugge. From 2002 to 2007 he was a Researcher at the Julius Centre for Health Sciences and Primary Care at the University Medical Centrer Utrecht, where he completed his thesis entitled 'Screening for type 2 diabetes in general practice'. In 2007 he was appointed an academic staff member in the Department of Guideline Development and Research Policy of the Dutch College of General Practitioners.
1. Wild S, Roglic G, Green A, et al., Diabetes Care, 2004;27:1047-53

2. Borch-Johnsen $K$, Lauritzen $T$, Glümer $C$, Sandbaek $A$, Diabet Med, 2003:20:175-81.

3. American Diabetes Association, Diabetes Care, 2004;27 (Suppl. 1):S11-S14.

4. Alberti KG, Zimmet P, Shaw J, Diabet Med, 2007:24:451-63.

5. Rutten GEHM, De Grauw WJC, Nijpels G, et al., Huisarts Wet, 2006;49:137-52.

6. Lauritzen T, Griffin S, Borch-Johnsen $\mathrm{K}$, et al., Int J Obes Relat Metab Disord, 2000;24:S6-S11

7. Ruige JB, de Neeling JND, Kostense PJ, et al., Diabetes Care 1997;20:491-6.

8. Alberti KG, Zimmet PZ, Definition, Diabet Med, 1998;15:539-53.

9. Janssen PG, Gorter KJ, Stolk RP, Rutten GE, Fam Pract, 2007;24:555-61.

10. Janssen PG, Gorter KJ, Stolk RP, Rutten GE, Scand J Prim Health
Care, 2008;26:160-65.

11. Janssen PG, Gorter KJ, Stolk RP, Rutten GE, Prim Care Diabetes, 2007;1:69-74.

12. Janssen PG, Gorter KJ, Stolk RP, Rutten GE, Br J Gen Pract, 2009;59:43-8.

13. Janssen PG, Gorter KJ, Stolk RP, et al., BMC Fam Pract, 2008:9:67.

14. Baan CA, Poos MJJC, Bilthoven: RIVM. Available at: uww.rivm.nl/vtv/object_document/01260n17502.htm (accessed 14 September 2006).

15. Christensen JO, Sandbaek A, Lauritzen T, Borch-Johnsen K, Diabetologia, 2004;45:1566-73.

16. Wilson JMG, Jungner G, Principles and Practice of Screening for Disease, Geneva: World Health Organization, 1968.

17. Unwin N, Shaw J, Zimmet P, Alberti KG, Diabet Med, 2002;19:708-23.

18. Coutinho M, Gerstein HC, Wang Y, Yusuf S, Diabetes Care,
1999:22:233-40

19. Sandbaek A, Griffin SJ, Rutten G, et al., The ADDITION study, Diabetologia, 2008;51:1127-34

20. Gaede P, Vedel P, Parving HH, Pedersen O, Lancet, 1999;353:617-22.

21. Gaede P, Vedel $P$, Larsen $N$, et al., N Engl I Med, 2003;348:383-93

22. Thoolen BJ, de Ridder DT, Bensing JM, et al., Diabetes Care, 2006;29:2257-62.

23. Unwin N, Shaw J, Zimmet P, Alberti KG, Diabet Med, 2002;19:708-23.

24. De Vegt F, Dekker JM, Ruhe HG, et al., Diabetologia, 1999;42:926-31.

25. DECODE Study Group, BMJ, 1998;317:371-5.

26. Barrett-Connor E, Ferrara A, Diabetes Care, 1998;21:1236-9.

27. Monnier L, Colette C, Dunseath GJ, Owens DR, Diabetes Care, 2007;30:263-9. 